\title{
Citizens' Opinions About a Digital Health Insurance Record
}

\author{
George KOSTIKIDIS ${ }^{\mathrm{a}, 1}$, Parisis GALLOS ${ }^{\mathrm{a}}$, Ioannis S. TRIANTAFYLLOU ${ }^{\mathrm{a}}$ \\ and Vassilis PLAGIANAKOS ${ }^{\text {a }}$ \\ ${ }^{a}$ Department of Computer Science and Biomedical Informatics, \\ University of Thessaly, Lamia, Greece
}

\begin{abstract}
An Electronic Health Insurance Record (EHIR) could give all the information needed to the insured citizens, informing them about the history of benefits and the health expenses. The aim of this work is to evaluate a Digital Health Insurance Record system as well as to explore the benefits of using this system, both for society and for each citizen individually. A quantitative survey was carried out using a questionnaire shared among 180 people in Greece in 2019. The questionnaire consisted of 25 closed-ended questions, 3 of which related to demographics and the remaining 22 related to the use and benefits of use of the EHIR system. Most of all people who took part in this study believe that EHIR can contribute positively giving both social benefits and benefits for the patients. An important finding of the study is the concern expressed by respondents about the security of the system in the management of sensitive personal data. Based on citizens' opinions a Digital Health Insurance Record can provide a lot of benefits to citizens and to the society as well as to the national health insurance system.
\end{abstract}

Keywords. Health Insurance Record, Evaluation Study

\section{Introduction}

Most of the times, the access to Health Insurance data can be done through the Electronic Health Records [1], providing sufficient but not all the data regarding the healthcare provision [2]. An Electronic Health Insurance Record (EHIR) could give all the information needed to the insured citizens, informing them about the history of benefits and the health expenses for which they have received compensation [3]. The purpose of this work is to evaluate a Digital Health Insurance Record system as well as to explore the benefits of using this system, both for society and for each citizen individually.

\section{Methods}

In order to achieve this objective, a quantitative pilot survey was carried out using a selfdeveloped questionnaire based on previous related studies [4]. The questionnaire was shared, in paper form, among 180 randomly selected people who had visited healthcare services in 3 cities (Kozani, Thessaloniki and Lamia) in Greece in 2019. The

${ }^{1}$ Corresponding Author, George Kostikidis, Student at the Department of Computer Science and Biomedical Informatics, University of Thessaly, Lamia, Greece; E-mail: kostikidis1@gmail.com. 
questionnaire consisted of 25 closed-ended questions, 3 of which related to demographics and the remaining 22 related to the use and benefits of use of the EHIR system. More specifically, 9 were bivalent and the 13 "Five Likert" scale. About the reliability of the tool Cronbach's A was 0.904 . Descriptive statistics and corellations between the participants' opinions about the EHIR and their personal characteristics were examined using SPSS.

\section{Results and Discussion}

The results of this preliminary survey show that the largest proportion of participants were young adults and middle-aged (18-55 years old are the $84,8 \%$ of the sample). Their level of education was also quite high (71,1\% completed high education studies), whereas in terms of their professional activity the majority consisted of public/private employees $(54,4 \%)$ and students $(22,2 \%)$. Regarding knowledge of the EHIR, respondents present an evenly distributed picture (yes $=48,3 \%$ ). Unfortunately, less than $20 \%$ of the participants use the system. Most participants $(81,7 \%)$ seem to have all the necessary resources to access and use the EHIR. The majority of the participants believe that EHIR can make a positive contribution by both social benefits and benefits for the patient. An important finding is the concern expressed by respondents about the security level of the system in the personal data management. Comparing the responses about the security with participants' age, it is found to be significant $(p=0,005)$ with correlation coefficient $-0,212$. All the other tested hypotheses were not confirmed.

\section{Conclusions}

According to the aforementioned results, citizens need to be informed properly about the EHIR system and the data security of this system. Based on citizens' opinions, a Digital Health Insurance Record can provide a lot of benefits to citizens and to the society as well as to the national health insurance system too. Under this frame, a dissemination of the system is recommended, because the majority of the citizens have positive opinions about the system but few are use it. Future work may include a wider scale survey, based on this pilot study, after the dissemination of the system, in order to record more accurately the citizens' opinions about a digital health insurance record system.

\section{References}

[1] Hatch B, Angier H, Marino M, Heintzman J, Nelson C, Gold R, et al. Using electronic health records to conduct children's health insurance surveillance. Pediatrics 2013;132(6);e1584-e1591.

[2] Heintzman J, Marino M, Hoopes M, Bailey SR, et al. Supporting health insurance expansion: do electronic health records have valid insurance verification and enrollment data?. Journal of the American Medical Informatics Association 2015; 22(4): 909-913.

[3] Petmesidou M. Challenges to Healthcare Reform in Crisis-Hit Greece. e-cadernos CES 2019; 31.

[4] Tavares J, Oliveira T. Electronic health record patient portal adoption by health care consumers: an acceptance model and survey. Journal of medical Internet research 2016;18(3). 